\title{
Status Report for NEET In-Pile Sensor Irradiation Capabilities at TREAT
}

N. Woolstenhulme

C. Baker

J. Bess

D. Chapman

C. Jensen

C. Hill

D. Wachs

S. Wilson

September 2017

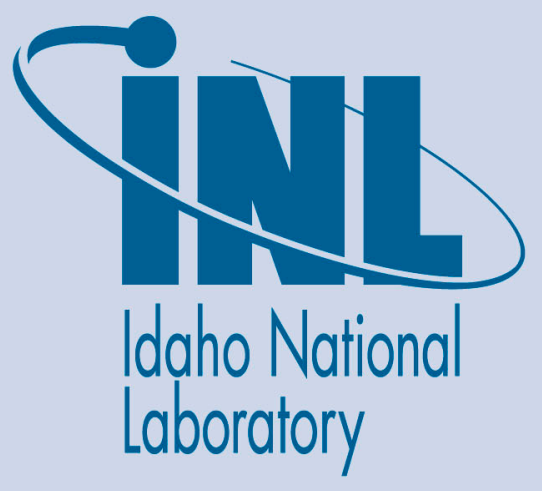

The INL is a U.S. Department of Energy National Laboratory operated by Battelle Energy Alliance 


\section{DISCLAIMER}

This information was prepared as an account of work sponsored by an agency of the U.S. Government. Neither the U.S. Government nor any agency thereof, nor any of their employees, makes any warranty, expressed or implied, or assumes any legal liability or responsibility for the accuracy, completeness, or usefulness, of any information, apparatus, product, or process disclosed, or represents that its use would not infringe privately owned rights. References herein to any specific commercial product, process, or service by trade name, trade mark, manufacturer, or otherwise, does not necessarily constitute or imply its endorsement, recommendation, or favoring by the U.S. Government or any agency thereof. The views and opinions of authors expressed herein do not necessarily state or reflect those of the U.S. Government or any agency thereof. 


\title{
Status Report for NEET In-Pile Sensor Irradiation Capabilities at TREAT
}

\author{
N. Woolstenhulme \\ C. Baker \\ J. Bess \\ D. Chapman \\ C. Jensen \\ C. Hill \\ D. Wachs \\ S. Wilson
}

September 2017

Idaho National Laboratory

Idaho Falls, Idaho 83415

http://www.inl.gov

Prepared for the

U.S. Department of Energy

Office of National Nuclear Security Administration

Under DOE Idaho Operations Office

Contract DE-AC07-05ID14517 


\title{
Status Report for NEET In-Pile Sensor Irradiation Capabilities at TREAT
}

\author{
INL/EXT-17-43274 \\ N. Woolstenhulme \\ C. Baker \\ J. Bess \\ D. Chapman \\ C. Jensen \\ C. Hill \\ D. Wachs \\ S. Wilson
}

Approved by:

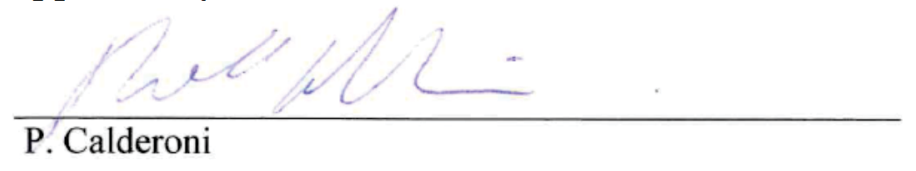




\section{CONTENTS}

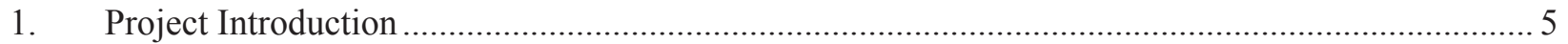

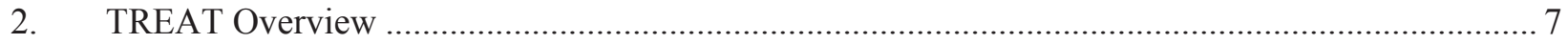

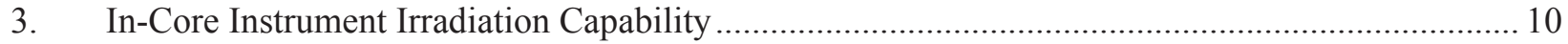

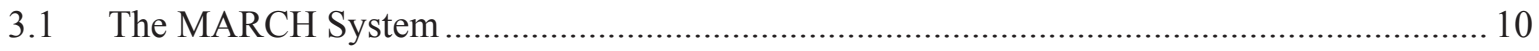

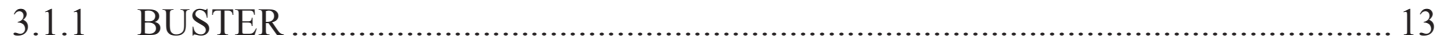

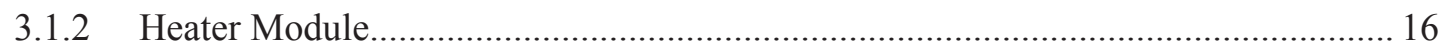

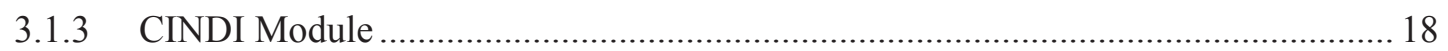

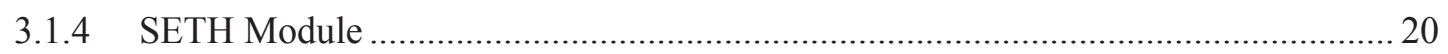

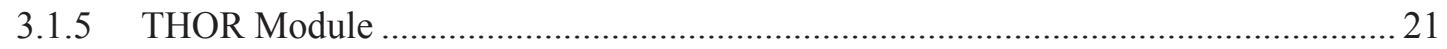

4. Preconceptual Design of Sensor Irradiations using MARCH .................................................. 24

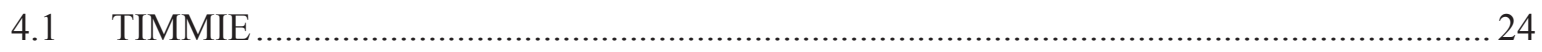

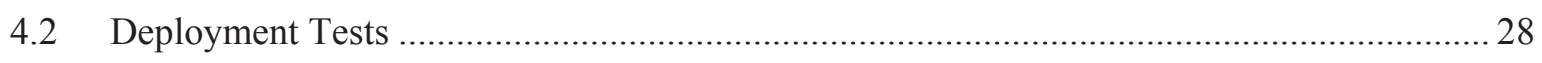

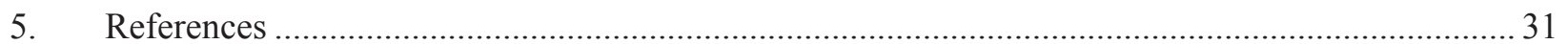




\section{Abstract}

The recently-established in-pile sensor development project under the Nuclear Energy Enabling Technology (NEET) program is focused on the development of measurement technologies to enable acquisition of data focused on the performance, properties, and boundary conditions of nuclear fuels/materials under irradiation in nuclear reactors. These sensors can be used in steady state irradiation tests to measure parameters of interest under conditions representing normal nuclear plant operation, or in transient irradiation tests to determine specimen performance under postulated unplanned conditions. In either case, development of suitable sensor technologies must include instrument irradiations to help guide sensor material/design selection, verify instrument performance, and demonstrate deployment viability. The Transient Reactor Test Facility (TREAT), while just a part of the overall capabilities portfolio needed to enable this sensor development mission, has unique capabilities and characteristics that make it well poised for a number of instrument irradiation data objectives including sensor survivability screening, response characterization, qualification tests, and in-situ properties measurements. TREAT's brilliantlybasic design, when married with appropriate irradiation hardware and analytic capabilities, fills a crucial capability gap between out-of-pile testing and irradiation in steady state high power research reactors. Leveraging a recently developed approach to irradiation testing in TREAT, the present work demonstrates that cost-effective and scientifically-impactful instrument irradiation capabilities for TREAT are a wellplaced capability in the NEET in-pile sensor development project. 


\section{Status Report for NEET In-Pile Sensor Irradiation Capabilities at TREAT}

\section{Project Introduction}

The recently established in-pile sensor development project under the NEET program is focused on the development of novel sensors, maturation of current measurement technologies, and adaptation of state-of-the art instruments in order to enable measurements focused on the performance, properties, and boundary conditions of nuclear fuels/materials under irradiation in nuclear reactors. Generally speaking, these irradiations can be categorized as either steady state or transient tests. Steady state irradiations typically make use of material test reactors to accumulate damage and measure data throughout experiment durations lasting months to years. Conversely, transient irradiations make use of transient test reactors where irradiations last seconds to minutes. While several test reactors have steady state and transient capabilities, reactors such as the Advanced Test Reactor (ATR) and TREAT are more strongly associated with steady state and transient irradiations, respectively.

Steady state irradiations are often intended to reveal performance phenomena and limitations of fuels/materials when everything is "proceeding as planned" at nuclear plants. As such, steady state irradiations are often focused on developing data which supports the economics of nuclear fuel applications. Transient testing, which is generally thought of as the study of nuclear fuel under powercooling mismatches, is typically meant to reveal performance under postulated unplanned conditions. As such, transient testing is often focused toward safety and regulatory data needs; in many cases making it of principle importance for advanced concepts seeking to license novel technologies for use. Steady state irradiations are also used to condition specimens for use in transient testing.

Both types of irradiation testing require in-situ data, and in some cases can employ common technologies. In other cases, these two types of irradiation can differ dramatically in their instrumentation needs. Development of steady state in-pile instruments has often fixated on the non-trivial problem of sensor survival under chronic exposure (e.g. fast neutron material damage). Transient instruments, on the other hand, must be developed to respond rapidly to and endure acute exposure from extreme nuclear and thermal conditions.

The motivation behind the work reported herein is to establish a foundational capability to facilitate instrument testing in TREAT (although it should be noted that some philosophic approaches could have relevance for other reactors). The link between a TREAT instrument irradiation capability and the development of transient instruments is quite natural. TREAT's characteristics, however, can also fill another capability gap for steady state instruments early in their maturation cycle before investment in long-term ATR or similar irradiations. Steady state reactors represent the end use of many in-pile sensor technologies, making the design/installation cost and immense irradiation durations an unavoidable circumstance where long-term neutron damage is a primary data condition. However, TREAT's lack of water and reactor pressure vessel greatly reduces the cost of instrument lead routing and experiment installation while unparalleled power maneuvering capabilities make TREAT apt for numerous sensor irradiation objectives. A more detailed description of the TREAT facility can be found in Section 7. An outline of TREAT-based instrument irradiation competencies is shown below:

- $\quad$ Screening survivability tests to determine the maximum nuclear heating/damage rates and material failure modes for sensor materials and instruments.

- These tests can help reveal phenomena behind reactor-induced sensor damage for material/concept screening both for novel sensors and evaluating available technologies for use in subsequent transient tests (e.g. TREAT capsules and loops).

- $\quad$ Noise \& response characterization to determine the response range for nuclear instruments and quantify "nuclear noise" in other instruments.

- This objective is particularly relevant for nuclear detectors (steady state or transient) owing to TREAT's power maneuvering capabilities ranging over several orders of magnitude. 
- These types of tests may also be used to quantify spurious nuclear effects on instruments (steady state or transient) meant to measure other parameters (e.g. temperature, pressure, displacement, etc.).

- Qualification tests to demonstrate that the instrument is ready to make its intended measurements in a nuclear environment, often using surrogates to reduce test cost.

- TREAT's ability to heat neutron-absorbing non-fueled materials to the range of interest for temperature measurements, for example, can enable relevant nuclear-heated instrument qualification tests to be performed with hardware that could be assembled in non-radiologic facilities.

- While TREAT cannot provide long term chronic irradiation damage, hybrid approaches may be sufficient in some cases. An example of this approach is storing an instrument in a TREAT capsule with a gamma source, and occasionally placing it in the core to make measurements and quantify chronic damage effects.

- Property measurements to further demonstrate the instrument's function by gathering in-situ data, typically on fuel specimens, which has innate scientific value beyond development and demonstration of the instrument itself.

- The relative ease in placing instruments and fuel specimens in TREAT enables property measurements under neutron/gamma bombardment (an under-addressed field in nuclear materials science).

- TREAT's capability package, including shielded hot cells at the neighboring Materials and Fuels Complex (MFC), circumvents the traditional problem of long-term instrument survival by outfitting highly-irradiated specimens prior to nuclear-environment property measurements in TREAT. 


\section{TREAT Overview}

TREAT was constructed in the late 1950's and provided thousands of transient irradiations before being placed in standby in 1994. More than two decades later, and following a successful effort to revitalize the facility, resumption of reactor operations at TREAT are both imminent and timely to address crucial nuclear fuel research data needs. TREAT is an air-cooled reactor driven by a core of graphite blocks having a small concentration of dispersed uranium oxide. Columns of these graphite-fuel blocks are hermetically encapsulated in zirconium alloy sheet metal canisters. Aluminum-sheathed unfueled graphite blocks are attached to the top and bottom of each fuel column; forming a discrete fuel assembly with $1.2 \mathrm{~m}$ of active core length. Along with control rod, experiment, and graphite reflector assemblies, these fuel assemblies are placed on a $19 \times 19$ gridplate with 361 available positions; creating a configurable core that can be adjusted to suit particular nuclear parameters or experimental objectives. A few fuel assemblies are typically removed from the central core positions to create a cavity for experiments. Experiment assemblies are typically removed from or placed into the core through a slot in the reactor's upper rotating shield plug, handled outside the reactor using shielded casks, and stowed below grade in storage holes when not in use. Four slots can be opened through the vertical concrete shield walls and permanent graphite reflector surrounding the above-grade core to provide various capabilities. Two slots are currently in use, one for a neutron radiography beam, and the other for a fuel motion monitoring system (commonly referred to as the hodoscope) capable of collecting spatiallyresolved data for fast neutrons born in experiment specimens. See Figure 1.

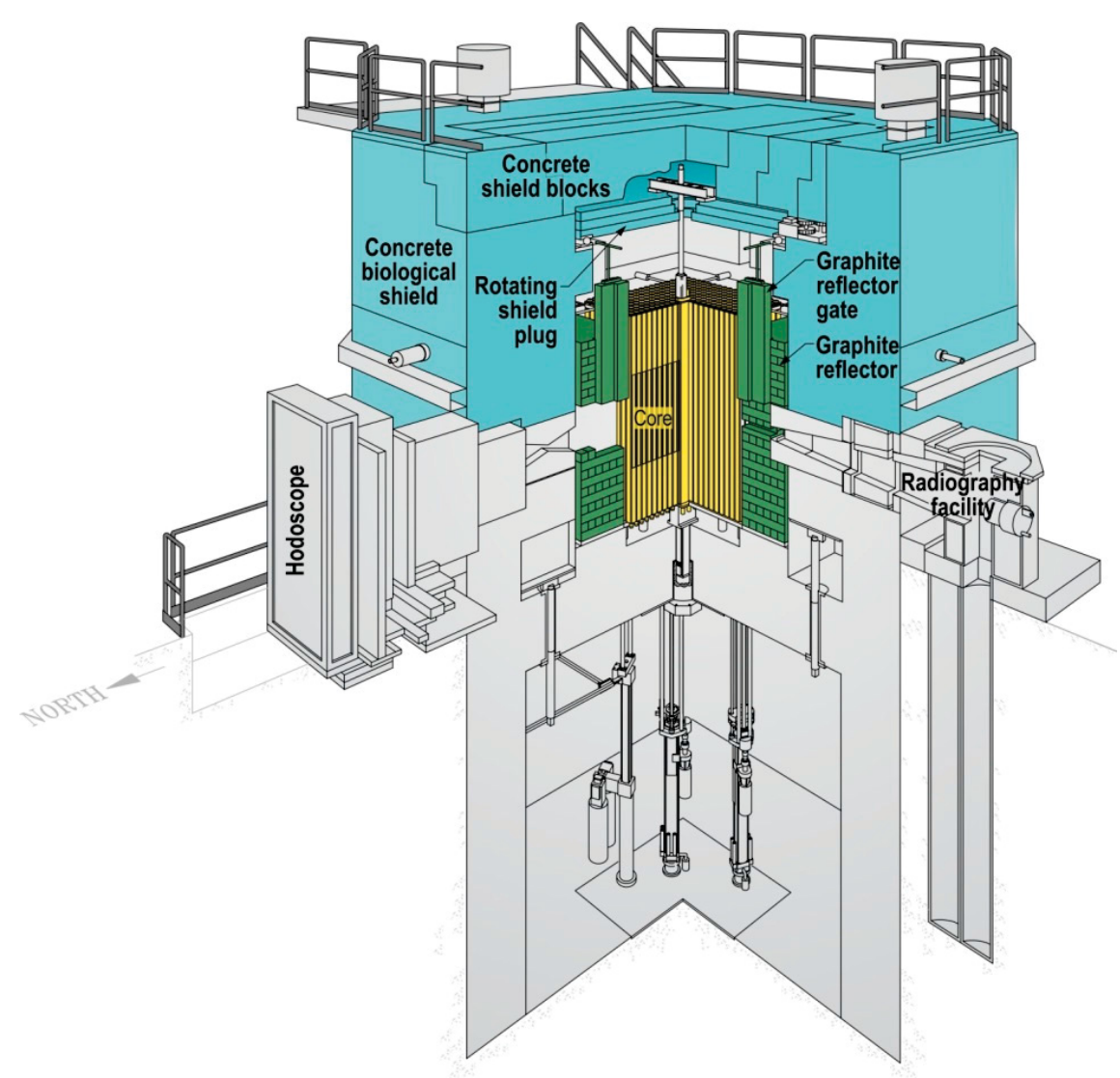

Figure 1: Overview of TREAT Features, $3 / 4$ Section View ${ }^{[1]}$

TREAT's air cooling system is adequate for steady state operation up to $120 \mathrm{~kW}$ thermal power useful for physics measurements, isotope build-in (e.g. $\left.{ }^{131} \mathrm{I}\right)$ for follow-on tests, neutron radiography, and other system checkout operations. TREAT's true capability, however, lies in its transient operations. TREAT's fast-acting hydraulically-driven transient control rods (see Figure 2) couple with the Automatic Reactor Control System (ARCS) to leverage the primarily-graphite core's negative temperature feedback 
to enable unrivaled power shaping capability. More than just a pulse reactor, TREAT is a transient reactor where inherently-safe core physics, a nimble transient rod drive system, and a philosophy of continual facility improvement work together to enable flexible power maneuvers relevant to current-fleet nuclear plants, advanced reactors, and scientifically-valuable power shapes.

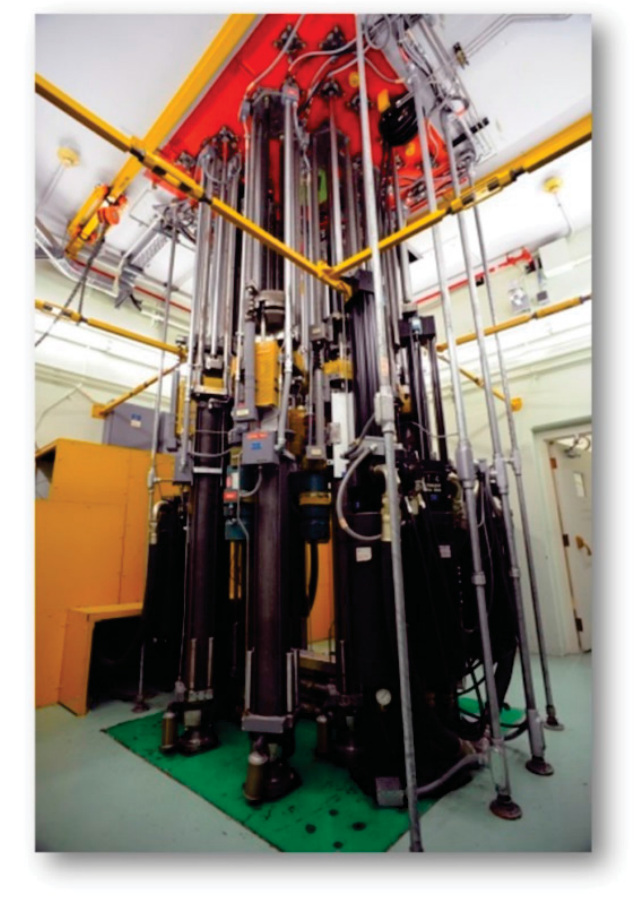

Figure 2: TREAT Control Rod Drive System Viewed from Subpile Room

TREAT's transients can be shaped to vary over several orders of magnitude in reactor power and transient duration; the precise shape being practically governed simply by the core energy capacity (currently $2500 \mathrm{MJ}$ ). ARCS can interface with experiment diagnostics instruments to enable feedback control and synchronization of experiment boundary conditions (nuclear, thermal hydraulic, etc.) Enhancements to core management/modeling methods and negative reactivity insertion "clipping" systems can augment this capability for transient capabilities transcending what is conventionally associated with graphite-based reactors.

Transient testing is typically thought of as the study of nuclear fuels and materials under events that are changing in instantaneous conditions. These events include myriad scenarios such as postulated offnormal, accidental, and upset conditions in nuclear plants. Among these scenarios, power-cooling mismatch conditions can challenge the integrity of fuel materials, making them the primary subject of nuclear fuel safety research. In-pile nuclear-heated transient testing, rather than out-of-pile testing (e.g. furnace testing), is typically required in scenarios where rapid power excursions, representative distributions of specimen heat generation, and coupled irradiation effects are needed to accurately represent behavior. TREAT's brilliantly-basic design, augmented by decades of incremental facility upgrades, create an extraordinary capability for nuclear-heated transient testing. TREAT's power maneuvering capability paired with experiment vehicles that provide specimen boundary conditions, complemented by in-situ instrumentation, and collocated with post-irradiation examination facilities, produces a full capability package able to address data needs for practically any reactor type or accident category. Example transient shapes that TREAT can currently provide are shown in Figure 3. 

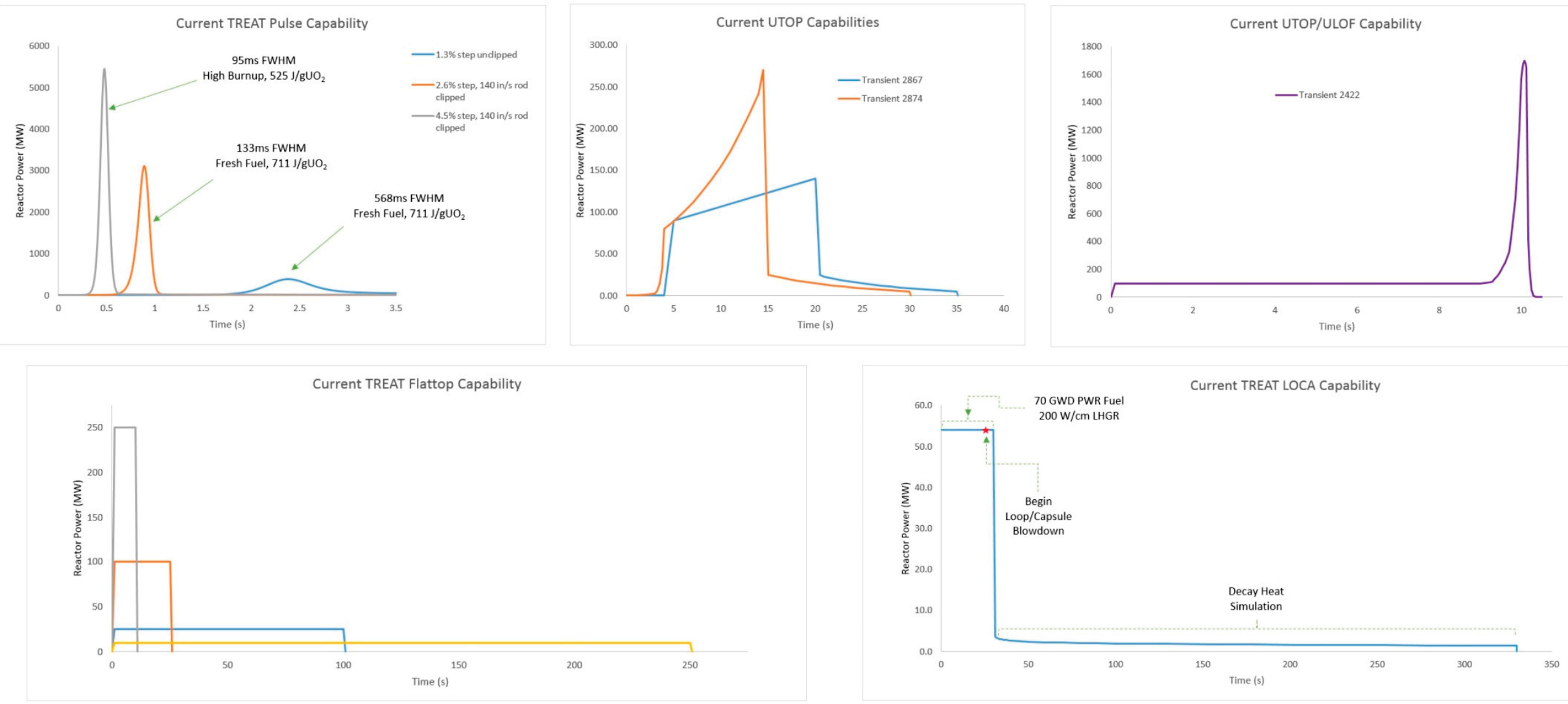

UTOP- Unprotected Transient Over Power

ULOF - Unprotected Loss of Flow

LOCA - Loss of Coolant Accident

Figure 3: Example TREAT Transient Shapes 


\section{In-Core Instrument Irradiation Capability}

It is possible to place sensors in multiple locations in or near the TREAT core, such as the numerous small coolant channels formed between the corner chamfers of four adjacent fuel assemblies or in peripheral positions (permanent reflector, bioshield, access slots). These instrument-irradiation positions can be utilized in situations where coolant channels' geometric constraints are not prohibitive $(\sim 15 \mathrm{~mm}$ square) or where flux levels are adequate (peripheral positions). In these situations varying levels of engineering control, configuration management, and safety evaluations will be required based on instrument-specific objectives and conditions, but implementation is generally thought to be much less onerous than the typical fueled TREAT experiment.

In other cases, instrument irradiations can require increased test geometry in the active core, involve potential effects of instrument material rearrangement needing to be mitigated by encapsulation, or entail measurements on nuclear-heated specimens that fall more into the realm of traditional TREAT fueled experiments. In these cases, which naturally represent a large fraction of irradiation needs for in-core fuelcentric sensors, specific core configurations and/or dedicated irradiation-supporting hardware is needed to create an instrument irradiation cavity. Some legacy TREAT hardware can be considered for this purpose, such as existing experiment calibration vehicles or non-standard TREAT fuel assemblies which have incore cavities (hodoscope slot assemblies, control rod assemblies), but would still likely require some special purpose adapter hardware to enable instrument irradiations. Deployment of new purposelydesigned instrument irradiation hardware and supporting capabilities, however, are more pragmatic in providing a broader-purposed and longer-lived solution for most, if not all, of the potential irradiation purposes described in Section 1.

The design solution described in this report is an application of a previously conceptualized irradiation vehicle system (described in Section 3.1) that enables several enhancements salient to instrument irradiations including the following:

- Ample test geometry in the high-flux central core position.

- Support for instrument lead routing.

- Compatibility with current TREAT experiment interfaces.

- Options for elevated temperature irradiation.

- Capability for test encapsulation when needed.

- Cost/schedule-reducing design philosophies.

- The basis for streamlined engineering and safety-based irradiation approvals.

\subsection{The MARCH System}

The latter half of TREAT's historic operation was best known for integral-scale testing of fuel specimens under postulated reactor plant accident conditions, but TREAT's underappreciated earlier history included simpler phenomena identification tests that elucidated fundamental behaviors and paved the way for these integral-scale tests ${ }^{[2]}$. Advances in modern computational capabilities and a resurgence of interest in novel reactor technology create an opportunity for emphasizing modernized science-based and separate effects test capabilities once again at TREAT. An innovative approach to this type of testing was conceptualized to leverage minor radioactivity built-in during brief TREAT irradiations by arranging small "fresh" specimens in low activation hardware so that they could be easily extracted and shipped for Post Irradiation Examination (PIE) within weeks. The concept was termed the Minimal Activation Retrievable Capsule Holder (MARCH) irradiation vehicle system and development began under INL Laboratory Directed Research and Development (LDRD) in Fiscal Year 2017 (FY17). Since then several projects, including the subject NEET in-pile sensor development project, have begun to interact with and support the MARCH team to build upon its foundational concepts while branching off to support development of specific modules, adaptations, and experiment objectives. The MARCH design philosophy is based on the following principles: 
1. Minimal Activation: Capitalize on a surprisingly large market segment of irradiation tests with specimens that are fresh (i.e. not pre-irradiated in another reactor), reasonably small, and not purposed for high levels of irradiation damage so that, with proper selection of materials and mechanical layout, personnel radiation exposure would be minimal, enabling PIE within weeks of irradiation.

2. An Exercise in Simplicity: Facilitate the science objectives of separate-effects, or limited combined-effects, irradiation tests by deemphasizing reactor-prototypic specimen boundary conditions (e.g. pressurized hot water, flowing liquid sodium, etc.) which represent sizeable engineering burdens or, in some cases, provide less-stable boundary conditions for post-transient data interpretation (e.g. coolant phase change).

3. Affordable Discovery through Modularity: Operating under the mantra of "TREAT is the nexus, MARCH is the gateway", employ common and reusable modules with cross-cutting capabilities in such a way that MARCH enables multiple missions at an affordable entry-point into transient science as an irradiation capability system, rather than a single-purpose irradiation vehicle.

The current MARCH system has several modules, with others envisioned, that provide various categories of capability. An overview of the MARCH system and its current modules can be seen in Figure 4. The following subsections describe each of MARCH's current modules. 


\section{The MARCH System}

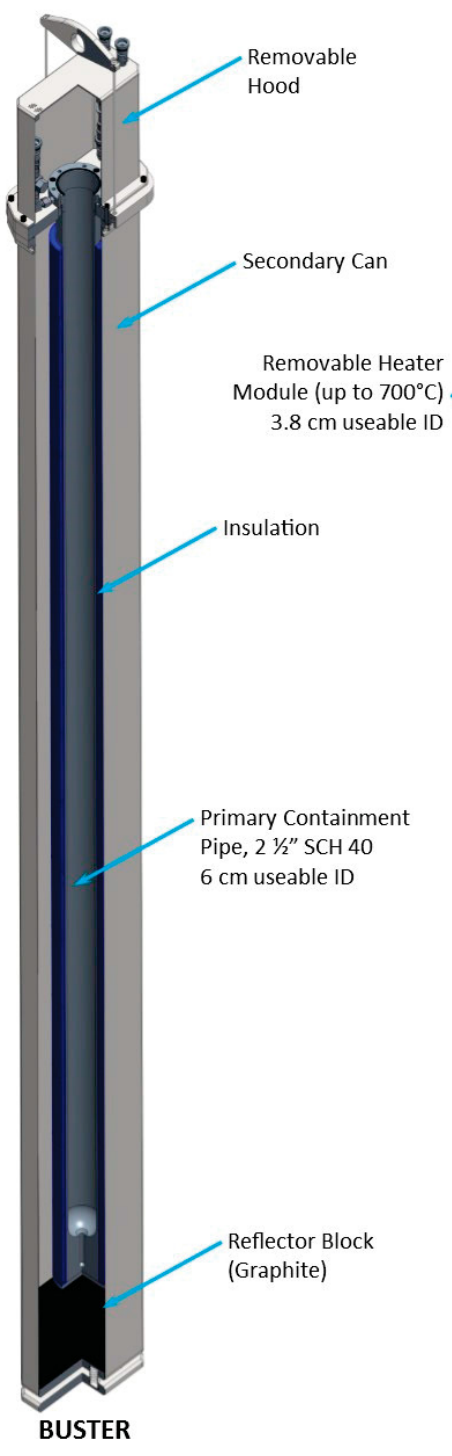

Multipurpose

Containment

Structure
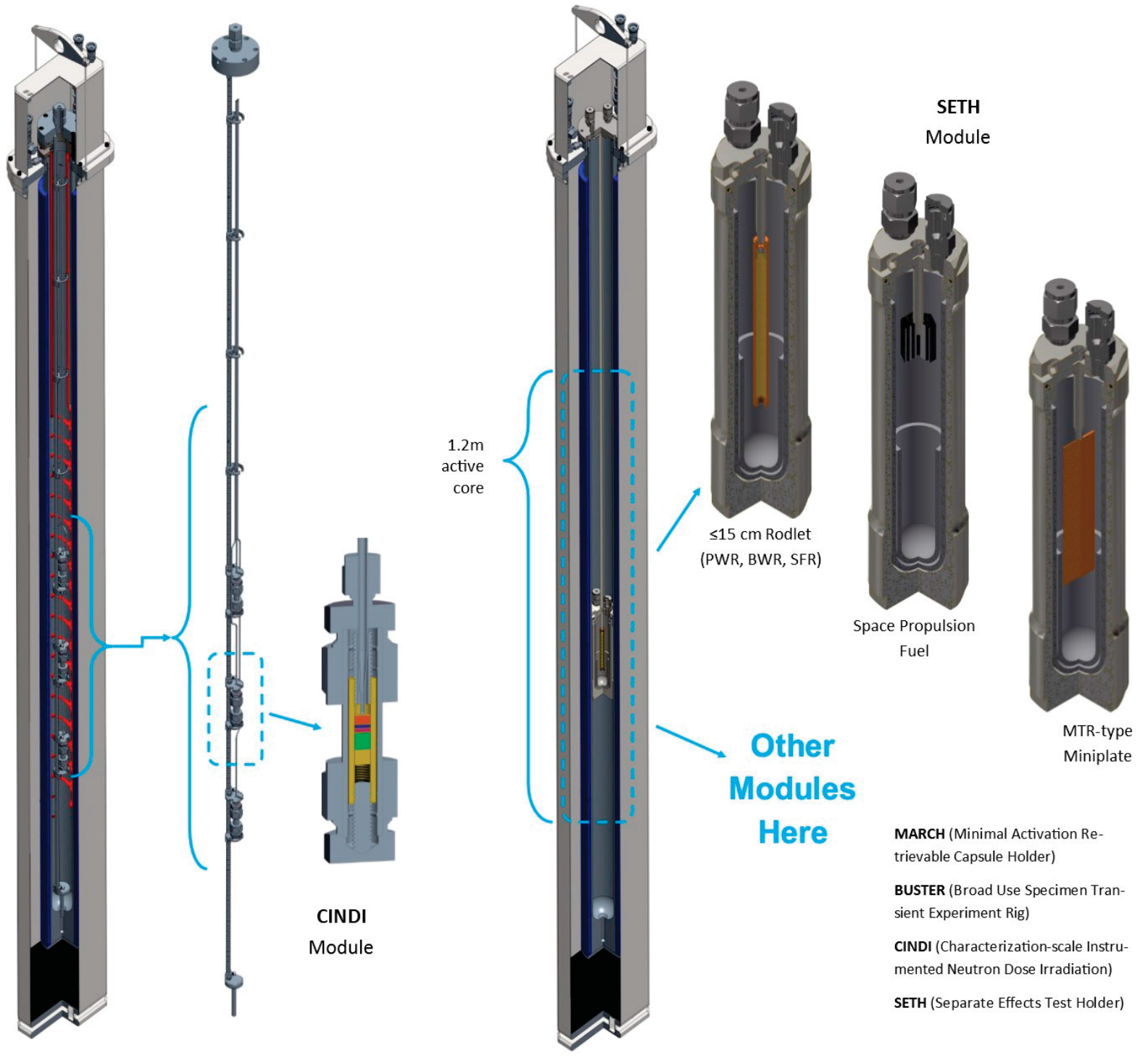

MARCH (Minimal Activation Re-

trievable Capsule Holder)

BUSTER (Broad Use Specimen Transient Experiment Rig)

CINDI (Characterization-scale Instrumented Neutron Dose Irradiation) SETH (Separate Effects Test Holder)

TREAT is the nexus, MARCH is the gateway

Figure 4: Overview of the MARCH System 


\subsubsection{BUSTER}

Since the reactor itself has no meaningful containment structure surrounding it (e.g. a reactor pressure vessel), TREAT experiments are required to provide their own containment to retain radiologic, chemical, and other energy-source hazards. The primary consideration for experiment safety is demonstrating that experiment containment is maintained while in the reactor during prescribed conditions and design criteria ${ }^{[3]}$. Among rules mandated by TREAT's safety bases pertaining to experiment containment, some stand out for their impact on the cost of TREAT experimentation. The MARCH system provides strategic solutions for satisfying these rules under some useful scenarios.

TREAT criteria require that containments provide a level of protection equivalent or greater than that afforded by the American Society of Mechanical Engineers Boiler and Pressure Vessel Code (ASME BPVC). The particular implementation of the ASME BPVC depends on experiment vehicle design, but typically results in limited selection of materials, permissible use conditions (e.g. temperatures), rigorous material procurement specifications, and extensive examination/testing requirements for fabricated components. These considerations ultimately drive TREAT experiment containments to be expensive items. Since transient testing conditions can render instrumentation inoperable and liberate contamination (especially for tests where fueled specimens are not clad, or where testing conditions breach cladding), it is common for structures containing instruments and specimens to be treated as single-use items. Owing to the unavoidable cost of the containment structure, a fundamental design philosophy which enables the MARCH system is to credit the safety containment function to a reusable stainless steel pipe structure, while the specimen and instrument support function is performed by a hermetic capsule-like module placed within it. In this way, the potentially single-use capsule is treated as a feature for convenience in handling, shipping, thermal management, and contamination control, but is not credited with the BPVC safety containment burden. This capsule-within-containment approach reduces the overall useable test volume, but is sufficient for MARCH's small-specimen mission.

In addition to making the potentially-disposable capsule more affordable, this approach also enables the modular concept in general and permits capsule modules to be constructed from less-conventional materials which activate less under neutron irradiation, notably including vanadium or high temperature use of titanium. During extraction, moderate-activation materials used in the containment structure (e.g. stainless steel) remain shielded in storage holes or stands.

The primary pipe provides approximately $6.0 \mathrm{~cm}$ of useable diameter over TREAT's $1.2 \mathrm{~m}$ active core. The pipe is surrounded by thermal insulation so that an electric heater module (see Section 3.1.2) can be installed for elevated temperature testing without causing overheat of adjacent TREAT fuel assemblies. The insulation is composed primarily of silica and silicon carbide in a microporous structure, which forms a rigid material that is machinable to tight tolerances. This type of insulation has a thermal conductivity an order of magnitude lower than ceramic fiber materials and enables large temperature gradients to be achieved in a minimal amount of space.

While the capsule module is not credited as the safety containment, it is designed to prevent contaminant leakage during expected testing conditions. As such, the primary pipe flange includes a gas sampling port to enable the internal environment to be analyzed for gross airborne contamination, confirming the integrity of the capsule module, prior to disassembly. This port is also used to fill the primary pipe with inert gas prior to irradiation so that high temperature exposure does not cause gross oxidation of material within. The pressure of the inert gas fill in the pipe will be no greater than atmospheric prior to heat up and irradiation. The pipe will be a closed system during irradiation. Owing to a large internal pipe volume, MARCH's small specimen mission, and lack of boilable coolant constituents within, the total pressure rise in the pipe due to transient heating will be minor compared to its pressure handling capacity. In this regard, it is expected that the pipe's containment capacity will maintain at least a $4 \mathrm{X}$ safety factor; a crucial cost-saving feature in enabling the majority of MARCHbased irradiations to receive approval on analytically-predicted nuclear heating alone without empirical determination of core-to-specimen power coupling factors in dedicated calibration campaigns.

In cases where plutonium-bearing or pre-irradiated specimens are used, two layers of containment are required even if safety analyses show that the breach of the primary is not credible. As a result, the primary pipe is surrounded by a stainless steel sheet metal canister roughly $10 \mathrm{~cm} \times 20 \mathrm{~cm}$ in-core cross 
section. The upper flange of the primary pipe hangs from a through hole in a large plate. A sheet metal hood can be fastened on top of this plate to complete the secondary containment. While the canister will always be used to provide the core geometry interface and protect the pipe insulation from damage, the hood is only required in cases where both layers of containment are needed. Compression seals in both the primary pipe flange and upper plate of the hood are used to hermetically route instrument and power leads through the top where support lines can be connected. Omission of the hood in permissible cases is a particularly relevant option for testing instrumentation where additional lead connectors are undesirable. The secondary canister provides handling interfaces and the ability for inert gas purge in the same fashion as historical TREAT experiments for compatibility with nearly the entire existing experiment infrastructure. Plumbing extension connections on the hood enable the gas purge to be used in either single or double containment mode.

Similarly, omission of the primary pipe's upper most blind closure flange may be permissible in some cases, such as testing of certain benign instruments, essentially turning the pipe into an interface for placement of test articles in the core, but not a containment structure (e.g. an opened-top "hole in the reactor"). This configuration may be permissible in cases where inner capsule modules are credited with the safety containment function or where specimens/hardware within cannot credibly rearrange, chemically react with air, and/or liberate contaminants. High temperature use of the heater module is be performed with the primary pipe closed and filled with inert gas to avoid gross oxidation.

All of these structures (primary pipe, insulation, secondary can, and secondary hood) have been jointly termed as the Broad Use Specimen Transient Experiment Rig (BUSTER); affectionately named since its mission is to both provide containment for those tests whose purpose is to bust fuel specimens and bust the myth that all irradiation testing is expensive. See Figure 5. The ability to operate BUSTER in a double-containment mode also enables it to be used for pre-irradiated specimens. In this scenario, capsule modules would be loaded with highly-radioactive specimens in a shielded structure, transported to TREAT, and placed into BUSTER using a series of shielding handling structures. This scenario entails a large radiation source negating the "minimal activation" philosophy, but some portions of the MARCH system philosophy could still be impactful for such tests. 


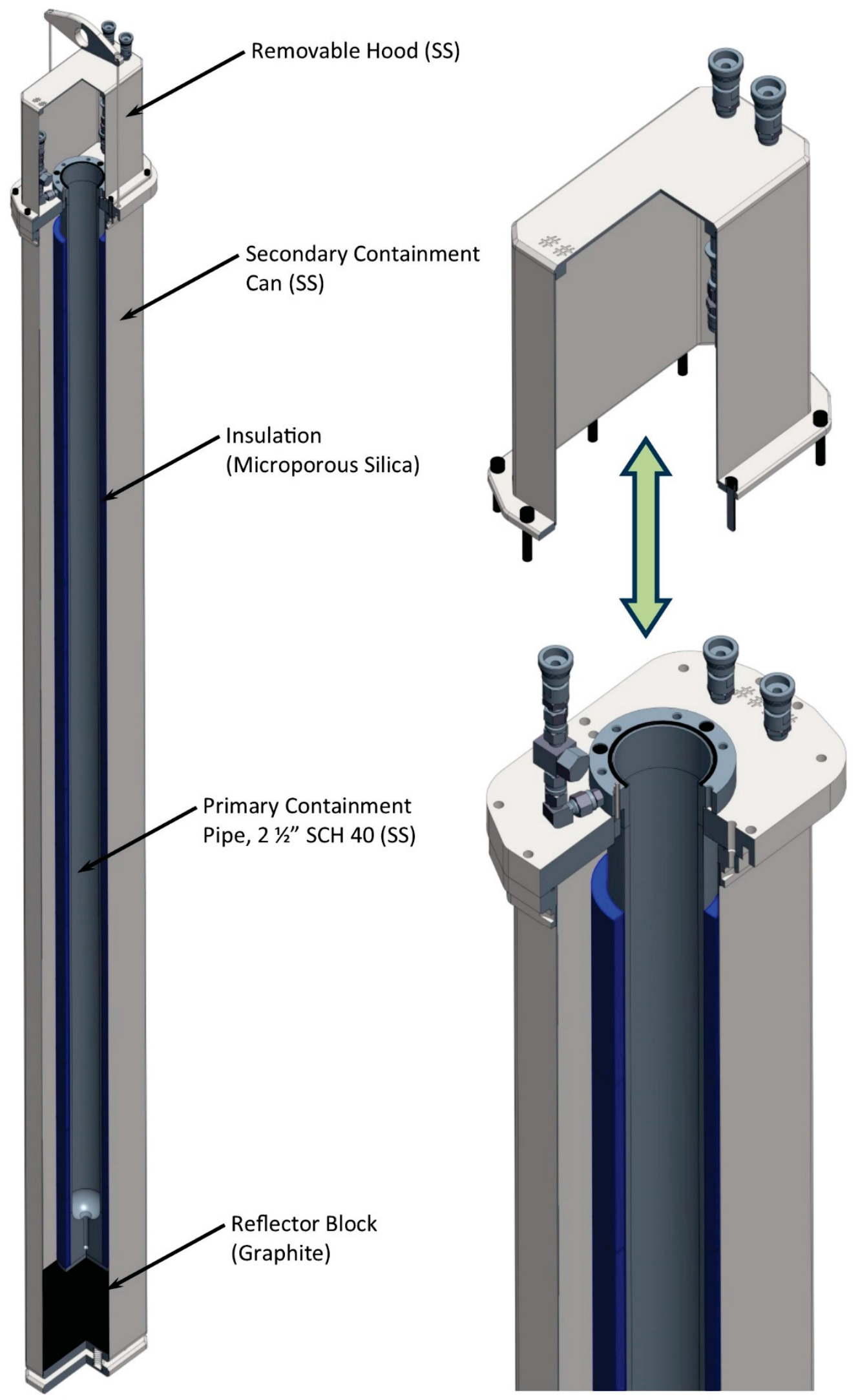

Figure 5: BUSTER Overview 


\subsubsection{Heater Module}

TREAT can nuclear heat fuels and neutron poisons to practically any desired temperature during brief transients. Still, the use of electrical heat is an important augmenting capability in establishing the desired material temperature before irradiation and for maintaining a desired temperature during longer lower power reactor operations. For this reason, the MARCH system was designed to be configurable with a heater module.

The heater module includes a tubular heater that is formed into a double helix shape that is sized to mate with the inner diameter of the pipe. Mineral insulated nickel-chrome resistance wire, all within a tubular Incoloy sheath, enable the heater module to operate up to $870^{\circ} \mathrm{C}$, although code-based limitations on the primary pipe restrict the heater temperature to $700^{\circ} \mathrm{C}$ pre-transient temperature. Transient nuclear heating and/or thermal insulation can then be used to achieve much greater specimen temperatures if desired. The heater is hermetically welded to and supported by a flange on top of the pipe assembly. Only the helically-wrapped section in TREAT's $1.2 \mathrm{~m}$ active core region is resistance heated so that, when combined with heat shield/insulation features above the hot zone, affordable polymer O-ring seals can be used at the upper flange. Since the heater materials are likely to become somewhat radioactive after multiple irradiations, the ring-shaped flange supporting it contains a hole through which experiment modules can be extracted. In this way, both BUSTER and the heater module can remain well shielded in below-grade storage holes while the experimental module is retrieved. A sheathed duplex thermocouple is attached to the tubular heater sheath near the top of the heated section to provide input to a programmable logic controller (PLC) for heater shutoff over-temperature protection. Temperature instrumentation in experiment modules, which varies based on the experiment, provides input to the heater control system.

There is a $3.8 \mathrm{~cm}$ useable inner diameter when the heater module is installed. Experiment modules which require additional space instead of high temperature operation, or which have electrical heating capabilities intrinsic to the experiment module, can elect to remove the heater module entirely. Depending on the decay time since its last use, heater module extraction can require shielding to minimize personnel dose. See Figure 6. 

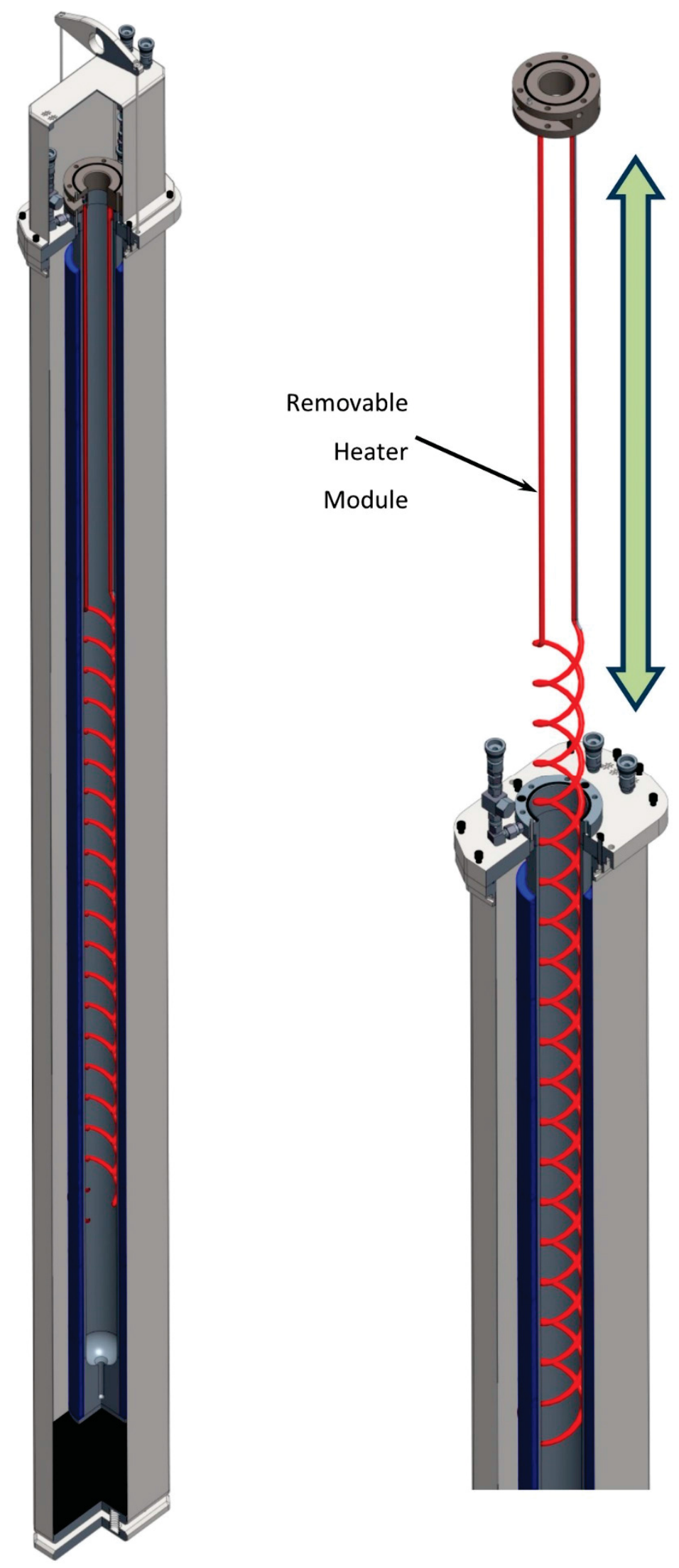

Figure 6: Heater Module Overview 


\subsubsection{CINDI Module}

A module termed the Characterization-scale Instrumented Neutron Dose Irradiation module (CINDI) is able to support the irradiation of small fuel samples or other specimens in well-controlled and monitored temperature conditions. CINDI capsules can house a small stack of disc-like fuel specimens $5 \mathrm{~mm}$ in diameter. One of each of the following specimen geometries, for example, fit in a single CINDI capsule:

- Laser flash specimen $(5 \mathrm{~mm}$ diameter $\times 2.5 \mathrm{~mm}$ length $)$

- Calorimeter specimen $(5 \mathrm{~mm}$ diameter $\times 1 \mathrm{~mm}$ length)

- Microscopy specimen $(5 \mathrm{~mm}$ diameter $\times 1 \mathrm{~mm}$ length)

- Thermal expansion specimen $(5 \mathrm{~mm}$ diameter $\times 5 \mathrm{~mm}$ length)

The capsules are compatible with established practices and gloveboxes for loading transuranic materials, enabling CINDI to house plutonium-bearing fuel compositions when BUSTER's secondary hood is installed. The overall CINDI module design is able to house three capsules simultaneously and supports them along a hanger rod with adjustable-height mounting brackets. In order to facilitate glovebox-based capsule assembly/disassembly schemes, the capsules are designed to be sealed by commercial Swagelok $^{\circledR}$ components. The majority of the physical hardware is constructed from titanium to reduce activation. A thin sleeve of vanadium (being both a chemically inert and very low activation material) surrounds the specimens to prevent titanium-specimen diffusion reactions during high temperature irradiations. CINDI irradiations can operate at up to $700^{\circ} \mathrm{C}$ temperature using the heater module. A single thermocouple penetrates into each capsule and measures the temperature of the uppermost specimen. When specimens of similar nuclear heating characteristics (e.g. fissile density) are grouped, this arrangement enables the as-run temperature history of each specimen to be acquired with reasonable accuracy so that it can be input to lower-length scale models while not overwhelming the capsule train with instrument leads. The axial position of capsules can be adjusted in order to use TREAT's axial flux profile in tuning nuclear heating rates. See Figure 7. 

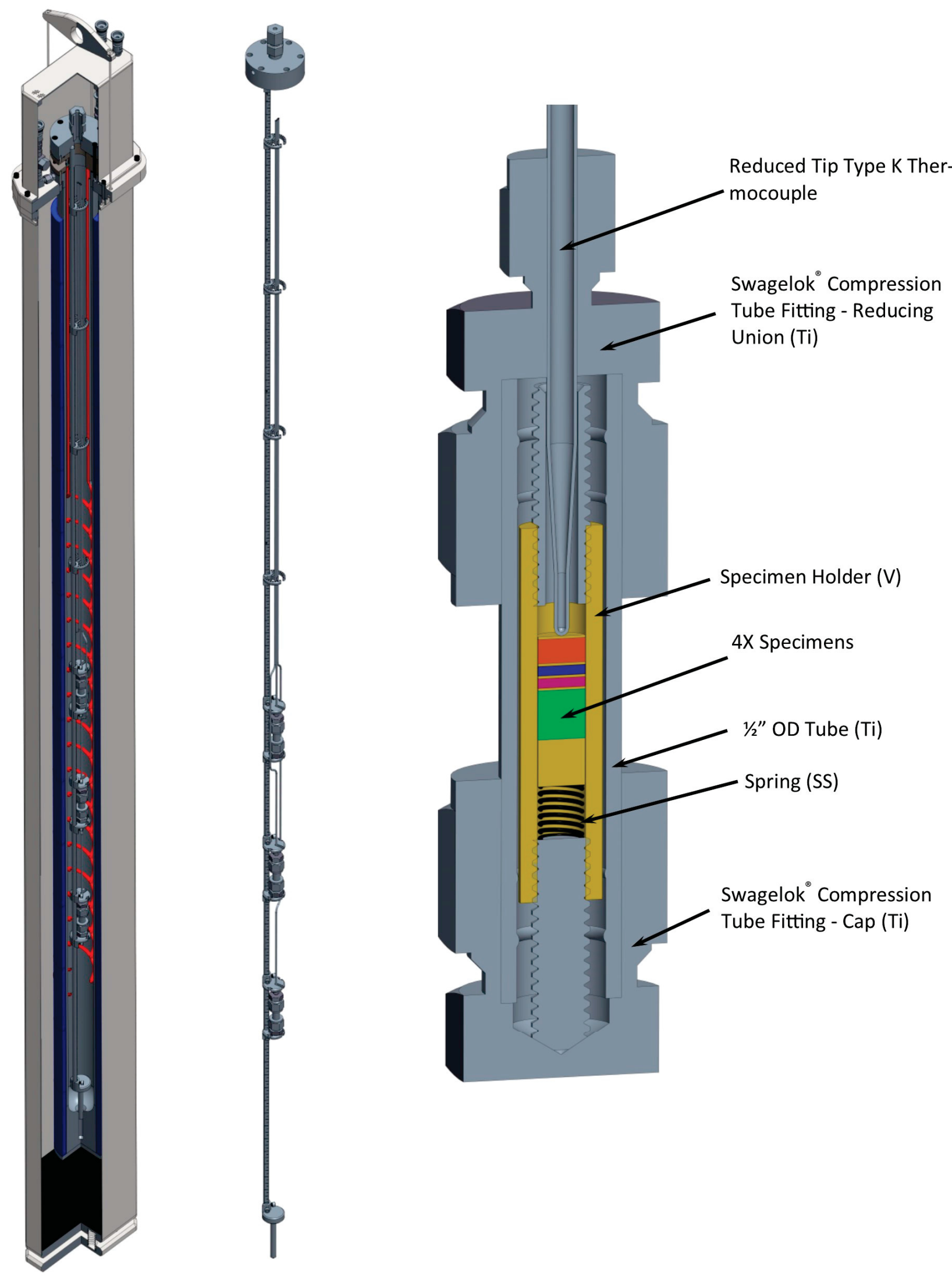

Figure 7: CINDI Overview 


\subsubsection{SETH Module}

A somewhat-larger specimen holder, made possible by removal of the heater module, can be used in the MARCH system while still falling within a reasonable radioactivity range. This module is termed the Separate Effects Test Holder (SETH) as shown in Figure 8.

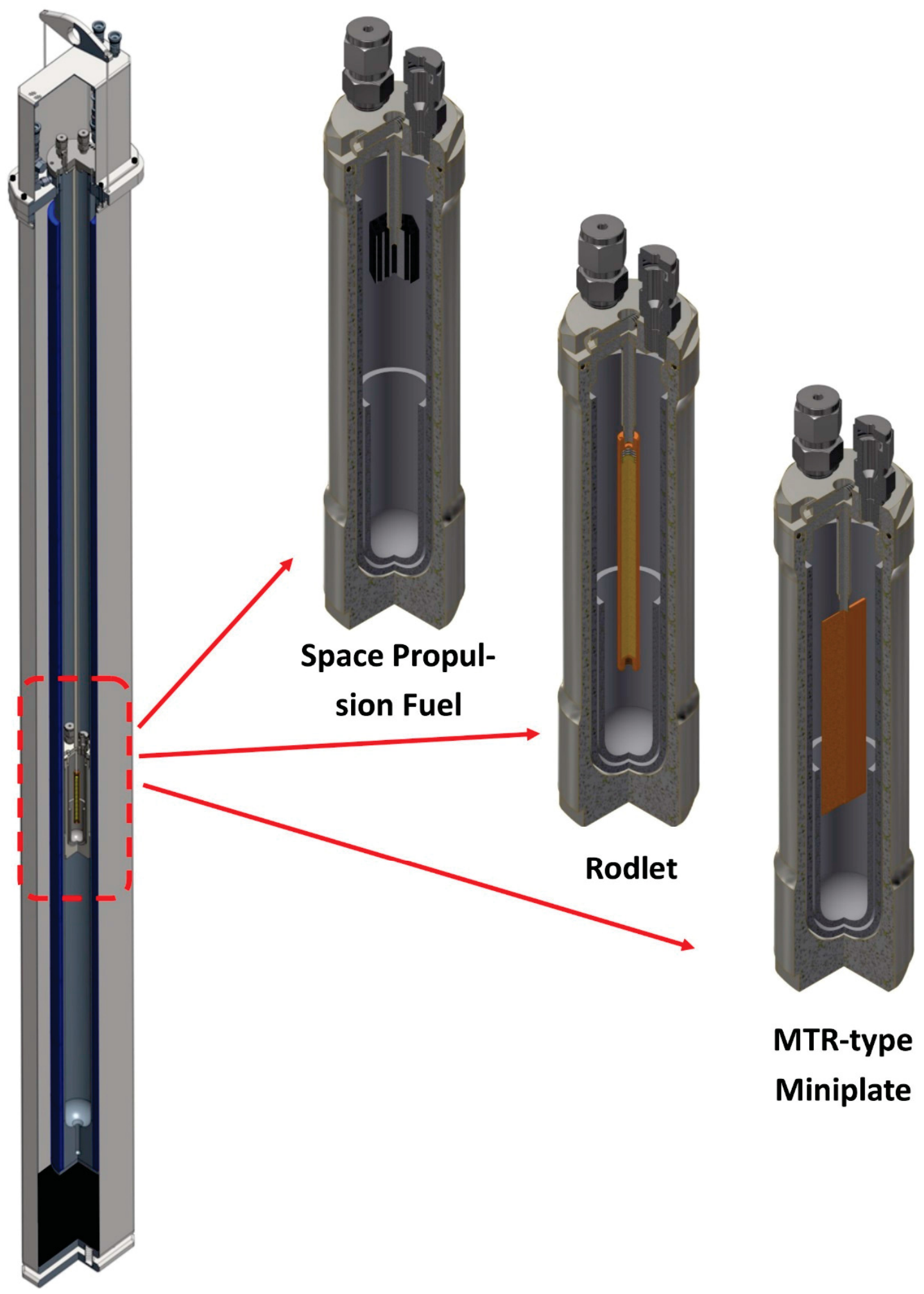

Figure 8: SETH Module with Example Fuel Specimens 
The larger internal volume in the SETH capsule design accommodates intermediate-scale fuel forms (rodlets, pins, plates, compacts) and provides room for fuel melt progression testing without geometric constraint from the capsule. The SETH module does not include the capability for prototypic reactor coolant conditions (e.g. hot pressurized water, liquid sodium) due to pragmatic engineering constraints. Rather, a reusable titanium capsule with off-the-shelf consumables (O-rings, instrument compression seals, and mullite crucibles) combine to create an affordable separate-effects and semi-integral scale testing platform. An inert gas fill provides a stable thermal boundary condition that minimizes heat loss during transient heating. When paired with the ability to accommodate more instrumentation around specimens, this approach makes the SETH module ideally suited for the following example types of tests:

- Brief pulse-like power excursions where specimen heat loss is minimal, such as phenomena identification studies on reactivity initiated accidents in water reactors.

- Fast nuclear-heated temperature changes to evaluate effects of thermal shock, such as poweron ramps for nuclear thermal propulsion fuels or prompt pulses for future transient reactor driver fuels (e.g. low enriched TREAT fuel).

- Severe accident melt down progressions scenarios where heat rejection to coolant is minimal such as phenomena identification studies on loss of coolant accidents in water reactors.

- Nuclear-heated tests purposed for comparison to electrically-heated out of pile accident tests such as nuclear-heated blister threshold temperature testing on material test reactor plates.

- In-situ property measurements or model development/verification separate effects tests where prototypic coolant conditions are not necessary, cost prohibitive, or a general nuisance in isolating and modeling phenomena.

The SETH concept is the basis for a variety of other irradiation projects, many of which build upon this foundational concept to support development of specific modules, adaptations, and experiment objectives. As a result, future SETH variants will likely be reconfigured, bifurcated, or enhanced in order to meet specific needs. Some potential enhancements to the SETH module have been suggested, discussed, and thought to have some merit including the following examples:

- A heater-wrapped version able to elevate starting temperatures to light water reactor coolant inlet temperature prior to irradiation, but filled with low pressure inert gas or steam (pressure vessel geometry constraints hamper the ability to achieve high pressure conditions).

- An enhanced in-situ instrument feedthrough version for advanced in-pile diagnostics and separate effects data.

- A room-temperature water-filled version which relieves internal pressure into the BUSTER pipe or auxiliary tank in a benign manner.

\subsubsection{THOR Module}

The SETH module was geared toward fuel testing by providing a large gas layer surrounding specimens making it well suited to rapid specimen energy injections and melt progression tests. Another module provides a different specimen thermal boundary condition with a heat sink for simulating fuel temperature response to slower overpower type transients. Ultimately, these types of transient simulations are best approached with fuel bundle testing in convective fluid loops (a capability clearly beyond the scope of the MARCH mission). However, historic work in TREAT suggested that a thick-walled heat sink capsule could be a particularly relevant capability for simulating the early stages of fuel performance under transient overpower conditions ${ }^{[4]}$. Building upon the MARCH system, another module referred to as the Temperature Heat-sink Overpower Response module (THOR) provides an analogous capability.

The THOR module makes use of a thick-walled titanium capsule as a heat sink. A clearance-fit hole houses a fuel pin with sodium in the annular gap. At operating temperatures, the sodium is molten and provides good heat conduction from the fuel's cladding to the titanium heat sink capsule. The required heat capacity in the capsule wall requires its outer diameter to be too large for insertion into the heater 
module. Instead, a small-diameter cable heater is wrapped around the capsule and the assembly can be placed within the BUSTER pipe.

This heater approach is not likely as reliable as the heater module design, which was designed for multiple irradiations at high temperature $\left(700^{\circ} \mathrm{C}\right)$, but should at least be adequate for achieving desired pre-transient temperatures $\left(100-400^{\circ} \mathrm{C}\right)$. This heater approach also requires that the irradiated heater be extracted along with the capsule. This is undesirable since the heater will contain moderately-radioactive materials after irradiation (Ni, Cr), but the heater mass is relatively small and these types of tests will likely include relatively large fuel specimens irradiated under longer shaped transients with appreciable total energy deposition. Except in cases where long cooling times are acceptable, the typical THOR fuel specimen will likely require some level of shielding to facilitate capsule extraction and shipping, making this heater scheme a viable approach. The same is true for some of the bulkier instruments comprised of stainless steel, nickel wires, etc. In this case, two Linear Variable Differential Transformers (LVDT) can be used to measure fuel and cladding elongation data on a rod with $\sim 20 \mathrm{~cm}$ fueled length. Additionally, commercial compression seals in the lid enable thermocouples to traverse into the capsule to measure fuel and cladding temperatures as needed. The THOR module enables transient overpower irradiations, obtains crucial data in-situ, and accelerates typical PIE for fuel concept screening, phenomena identification, and accident performance understanding of semi-integral scale fuel specimens. 


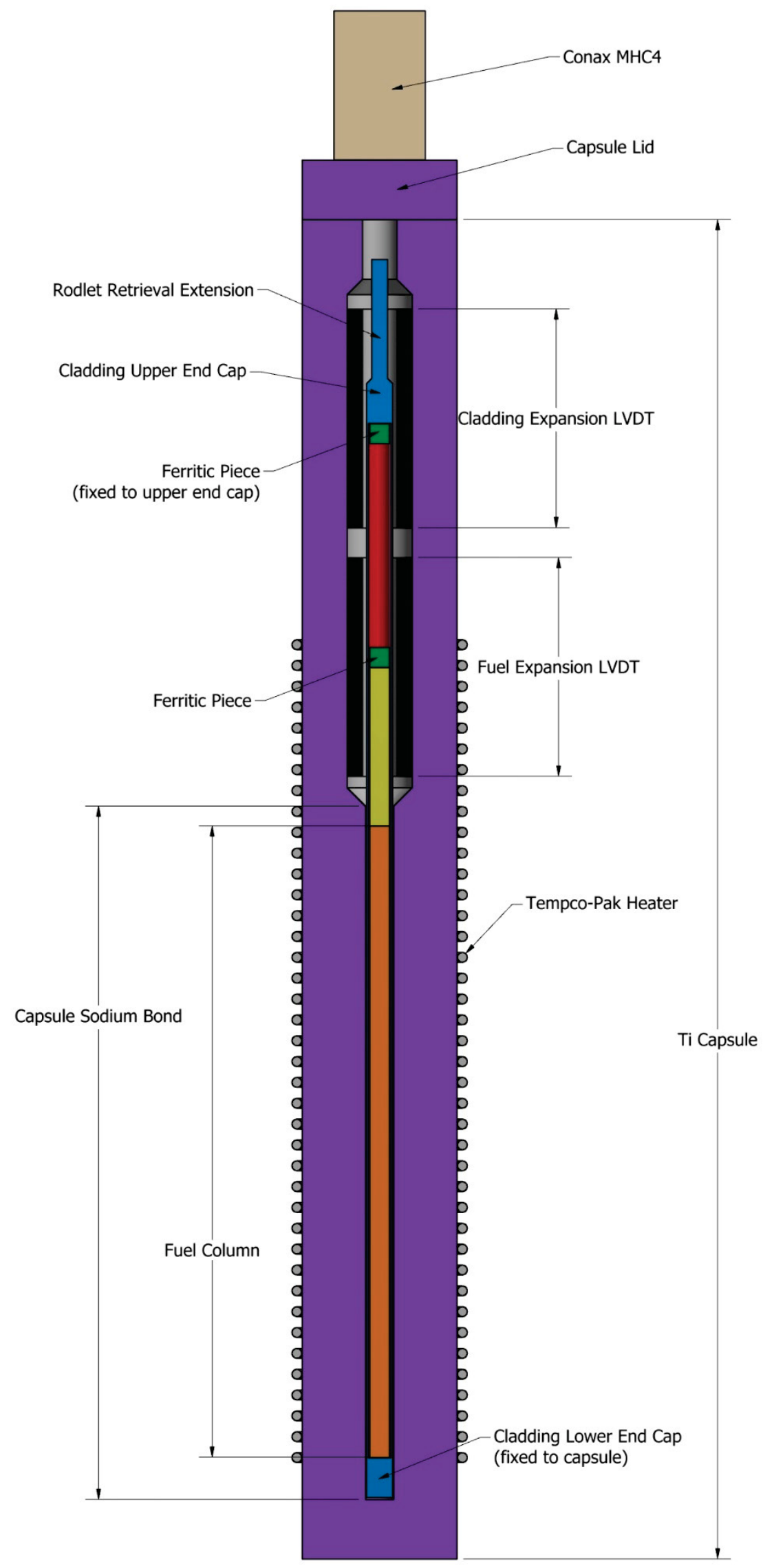

Figure 9: THOR Module Schematic 


\section{Preconceptual Design of Sensor Irradiations using MARCH}

Starting in the latter half of FY17, shortly after commissioning of the NEET in-pile sensor development program, design efforts commenced to set the foundation for a broadly-purposed TREAT instrument irradiation capability using the MARCH system. At the time this report was written, no definitive plans had been made for which instruments would be irradiated first in TREAT, although several relevant ideas were developing. As such, the present design work is considered preconceptual in nature, but owing to the relative maturity of the MARCH system and comparative ease in most instrument irradiations, the present work is established enough to illustrate how these future irradiation designs may be accomplished. These preconceptual designs provide context for the future brainstorming and innovations in the field of instrument irradiation testing for deployment.

\subsection{TIMMIE}

Two of the most impactful objectives for near-term instrument irradiations in TREAT, as described in Section 1, are survivability screening and noise/response characterization. Survivability tests can be used to determine the maximum acute exposure limits for instrumentation. These tests can help reveal phenomena behind reactor-induced sensor damage for material/concept prioritization both for novel sensors and available technologies. A straightforward application of these types of tests is irradiation of transient instruments, such as those being developed for use in future TREAT capsules or loops, to determine whether they can survive high temperature exposure, acute neutron gamma bombardment, and repeated exposure cycles. With one chance for success to provide their invaluable data, instrument selection for end use in fueled TREAT tests (which can involve heavy investment and years to prepare irradiated samples) can benefit greatly from these preceding instrument irradiations to screen candidate sensors and ensure survival. Similarly, instruments intended for steady state reactors can benefit from this approach to determine whether comfortable margin exists between planned sensor operating conditions and potential conditions arising from operational uncertainties and off-normal events.

Noise \& response characterization tests can determine the response time/range for instruments and quantify "nuclear noise". This objective is particularly relevant for nuclear detectors owing to TREAT's power maneuvering capabilities ranging over several orders of magnitude in flux levels. These irradiations may be used to determine sensor saturation points, identify calibration ranges, and guide signal processing strategies (e.g. determine flux levels for switching between pulse and current mode acquisition).

These types of tests may also be used to quantify spurious nuclear effects on instruments which are not meant to monitor nuclear parameters (e.g. sensors meant to measure temperature, pressure, displacement, etc.). These spurious effects can arise from nuclear sensor heating, atom displacement in sensors, photon interactions, acoustic events, and other elusive effects. Nuclear noise observations may be helpful in improving, or abandoning, a particular sensor technology. In other cases, irradiating instruments in the absence of the parameter they are meant to measure can quantify the repeatability and magnitude of spurious contributions in order to account for these effects in data interpretation. Similar approaches were used decades ago in TREAT to compare the response of various pressure transducers as shown in Figure 10. While the above-described survivability tests show whether instruments can endure irradiation, response characterization tests show whether instruments can provide reliable data in end use tests (a crucial consideration for data to be used in regulatory approvals). The natural similarity between both of these tests objectives enables them to be treated in combined experiments in many circumstances. 
TEST CONDUCTED ON UNBONDED STRAIN GAGE

TRANSDUCERS (Manufacturer C, Pickups Exposed

to Constant Pressure of 1 atm)

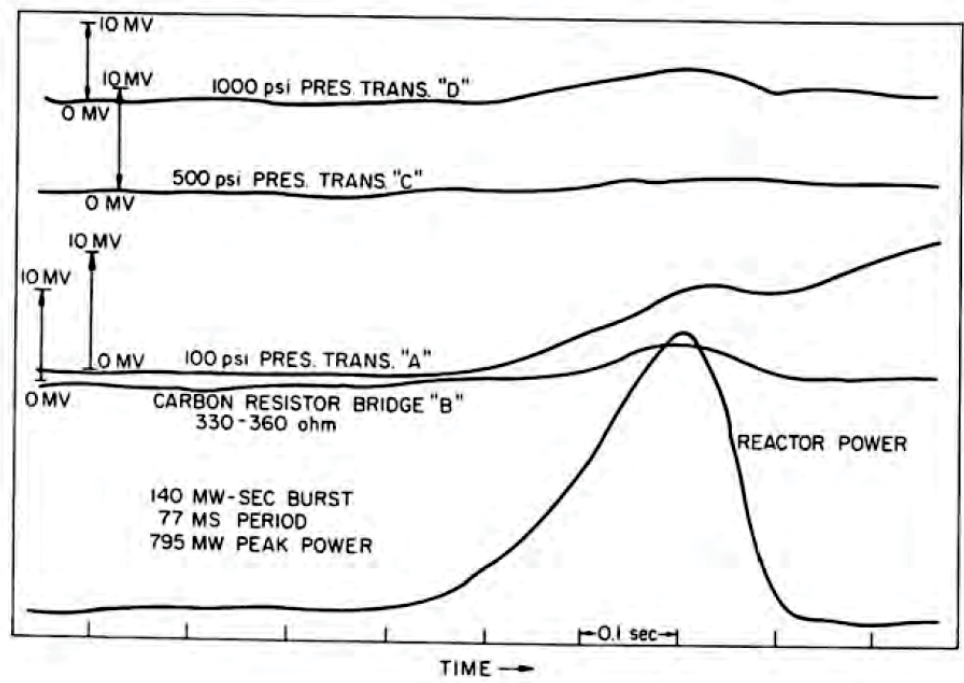

Figure 10: Pressure Transducer TREAT Irradiations ${ }^{[5]}$

These two data objectives naturally fall into the early stages of instrument maturation cycles and benefit most from the cost/schedule-reducing aspects of TREAT and the MARCH system. For this reason, a dedicated module concept paired with a safety/engineering envelope enables irradiation of instrumentation (and materials in general), to be performed as affordably as possible. This module, termed the Transient Instrument and Materials Maximum Irradiation Envelope (TIMMIE), includes reusable low-activation hardware inspired by the adjustable-height instrument mounting and lead-wire management features of the CINDI capsule holder. Like a building block system, TIMMIE accommodates various instrument form factors (as illustrated in Figure 11) to be placed in desired core positions using sliding mounting hardware, set screws, and an engraved ruler on the hanging rod. In a similar fashion, debris and melt-catching cups (not pictured) can be attached to the hanger rod to surround instruments which could potentially rearrange during irradiation. A commercial Conax ${ }^{\circledR}$ compression seal on the top flange enables exchanging of off-the-shelf sealant units to accommodate various combinations of lead-wire quantities and diameters.

Instruments small enough to fit within the heater module can be irradiated in TIMMIE with up to $700^{\circ} \mathrm{C}$ electrical preheat. This capability enables instruments to be exposed to in-core temperature conditions representing current-fleet and advanced-reactor plants while being bombarded by neutrons and gamma irradiation. The ability to control and measure internal temperature through a combination of electrical and nuclear heating can also be a used for irradiation of passive temperature instruments such as melt wires. Larger instruments can be irradiated without the heater module. 


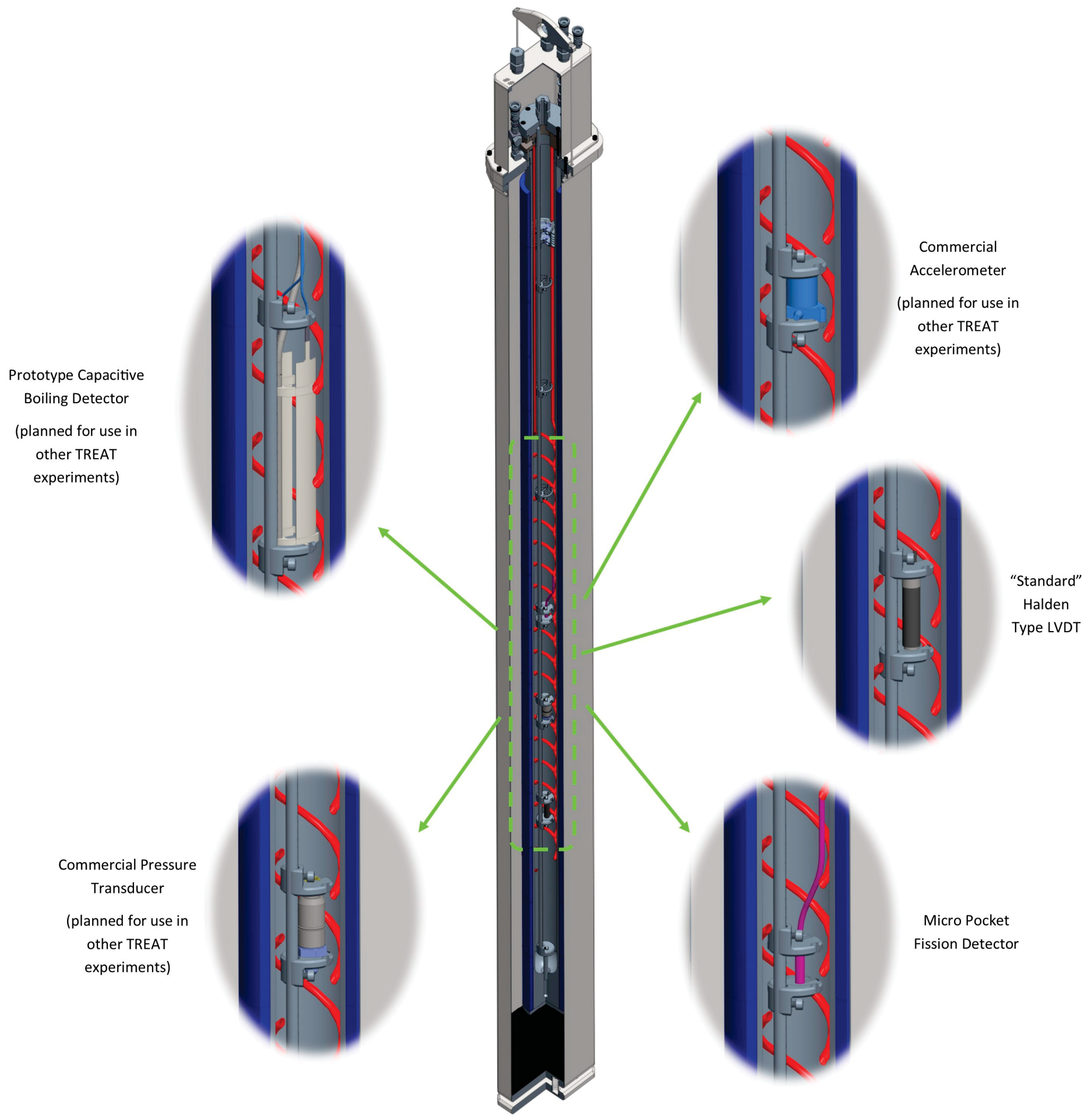

Figure 11: Example Instruments using TIMMIE in Heater Module

While TIMMIE includes some basic hardware, its true innovation is a safety analysis philosophy based on pre-populated nuclear/thermal parameters in a straightforward calculation tool. The TIMMIE analysis tool permits safe irradiation of pre-analyzed materials based only on their constituent masses; bypassing the cost of geometry-specific modeling. This transformative idea relies on some enabling assumptions so that enveloping nuclear parameters can be calculated. First, the core configuration is assumed in a particular arrangement of fuel, dummy, and hodoscope assemblies with BUSTER in the central position. Next, it is assumed that complicated nuclear interactions, such as fuel within proximity to 
moderators, will be addressed by geometry-specific models. These assumptions enable thin wires (minimal self-shielding) to be modeled for peak nuclear heat generation and isotope activation rates. Similarly, extreme geometric scenarios such as dense material spheres and homogenous dispersions can be modeled to determine the quantity that would cause a significant reactivity change $(0.05 \% \Delta \mathrm{k} / \mathrm{k}$, TREAT's threshold for requiring new core characterization processes). The elements and isotopes potentially found in instrumentation are all modeled in this way to build a database so that maximum irradiation parameters can be calculated (e.g. maximum constituent masses and core energy release).

This database is augmented by necessary thermal properties for common material compositions (ceramics, alloys, polymers, etc.) with the option for custom properties to be input for new materials. Finally, simple equations which relate TREAT's experiment safety energy release as a function of reactivity available in the transient rods enable computation of the maximum permissible irradiation parameters. Maximum thresholds are computed for adiabatic energy deposition to determine specimen melting and/or acceptable limits for the heat capacity of TIMMIE hardware. Once complete, the analytic tool will be available as a configuration-controlled spreadsheet, web-based tool, or similar application. This approach will allow users to enter instrument mass constituent information and obtain a printout of the permissible experiment limits nearly-instantly. Assuming that TREAT is available to perform the irradiation and that the instrument can be shown to conform to the parameters in the printout, this tool will greatly facilitate instrument irradiations in a way rivaled only by out-of-pile instrument testing. The philosophic goal underlying the TIMMIE analysis tool is to enable a summer intern to conceive, perform, and publish the results of an instrument irradiation before returning to coursework in the fall. See Figure 12 for an example illustration of the TIMMIE safety analysis tool.

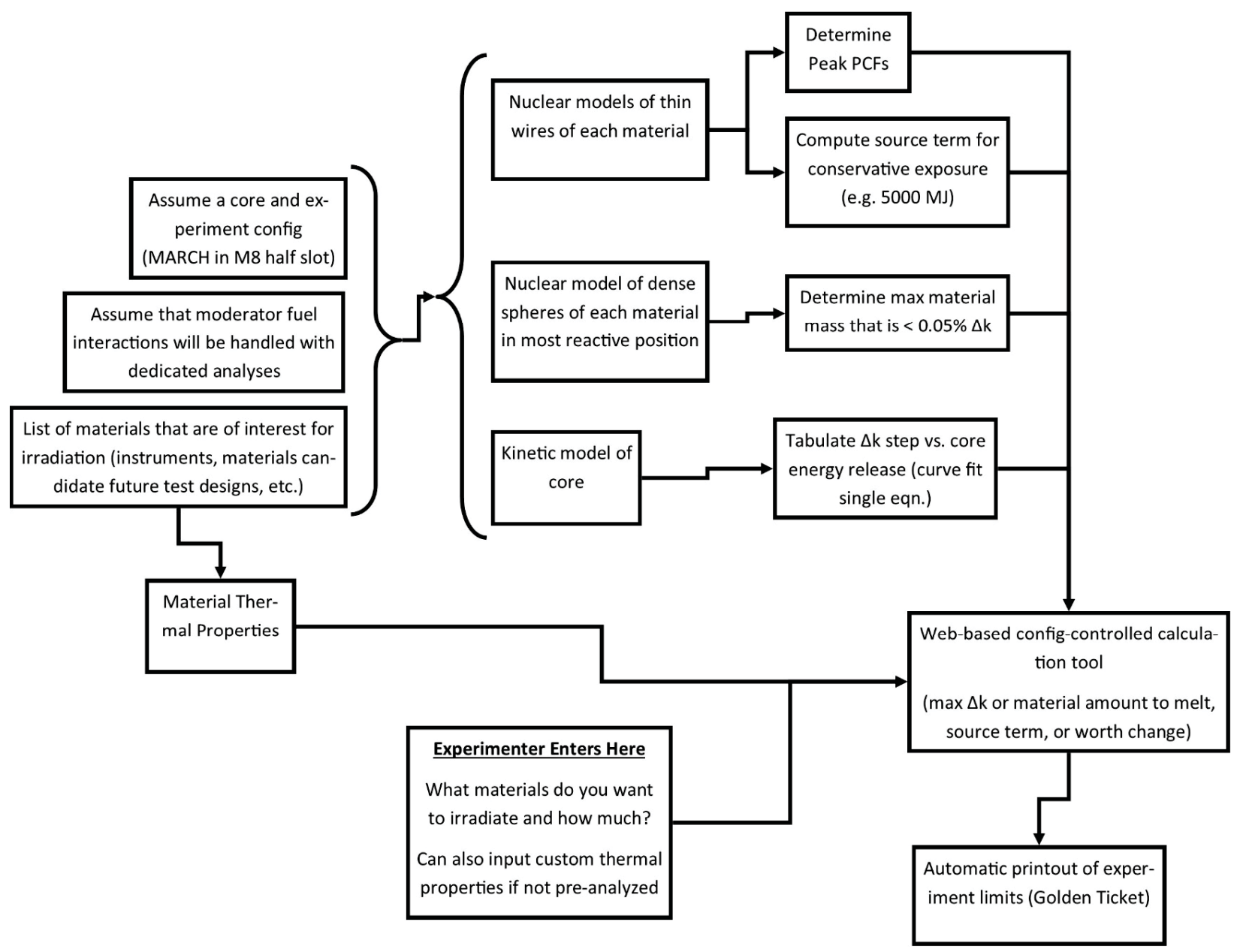

Figure 12: TIMMIE Analysis Tool Overview 


\subsection{Deployment Tests}

Other highly valuable TREAT-based instrument irradiation objectives, as described in Section 1, include qualification tests and property measurements. The fundamental difference between these two test objectives, and those described in section 4.1, is that they involve irradiation of instruments along with specimens or other means to incur changes in the parameters that the instrument is meant to measure. Naturally falling into the later stages of instrument maturation cycles, these irradiations treat sensors as an integral-system to demonstrate that they are sufficiently mature for end use in nuclear environments. These deployment tests may be viewed as the seminal event in an instrument's maturation under this NEET program; laying the groundwork for future adoption by fuel development programs, reactor plants, and other end users.

Specifically-focused qualification tests likely depart from the broadly-purposed TIMMIE module in many cases, increasing their required design and analysis resources by some amount, but foundational modules of the MARCH system can still be utilized. When surrogate specimens can be used, which enables test assembly in non-radiologic facilities, these tests objectives can still be accomplished in cost effective-ways. Several examples of surrogate-based qualification tests are illustrated in the lists and figures below:

- Borated metal alloy specimens (which can be heated to practically any desired temperature in prompt TREAT pulses) in the SETH capsule in the presence of temperature instrumentation (see Figure 13 for example layout).

- LVDT's in the THOR capsule where the ferritic cores are displaced by high thermal expansion metals (e.g. borated-aluminum) heated first by electrical preheat and then by nuclear heat.

- Placement of a fiber optic-based instrument in a SETH capsule, alongside a gamma source, with repeated cycles of placing it in the core to make measurements followed by residence in TREAT storage holes, to quantify gamma darkening effects on measurements made in nuclear environments.

- Connecting in-core pressure instrumentation to ex-core fluid lines so that fluctuations in pressure can be synchronized with irradiations and compared to ex-core instruments.

- Other electro-mechanical systems placed in MARCH modules that produce repeatable motion, vibration, load, strain, etc. so that comparison of measurements can be made against the same system out-of-pile. 


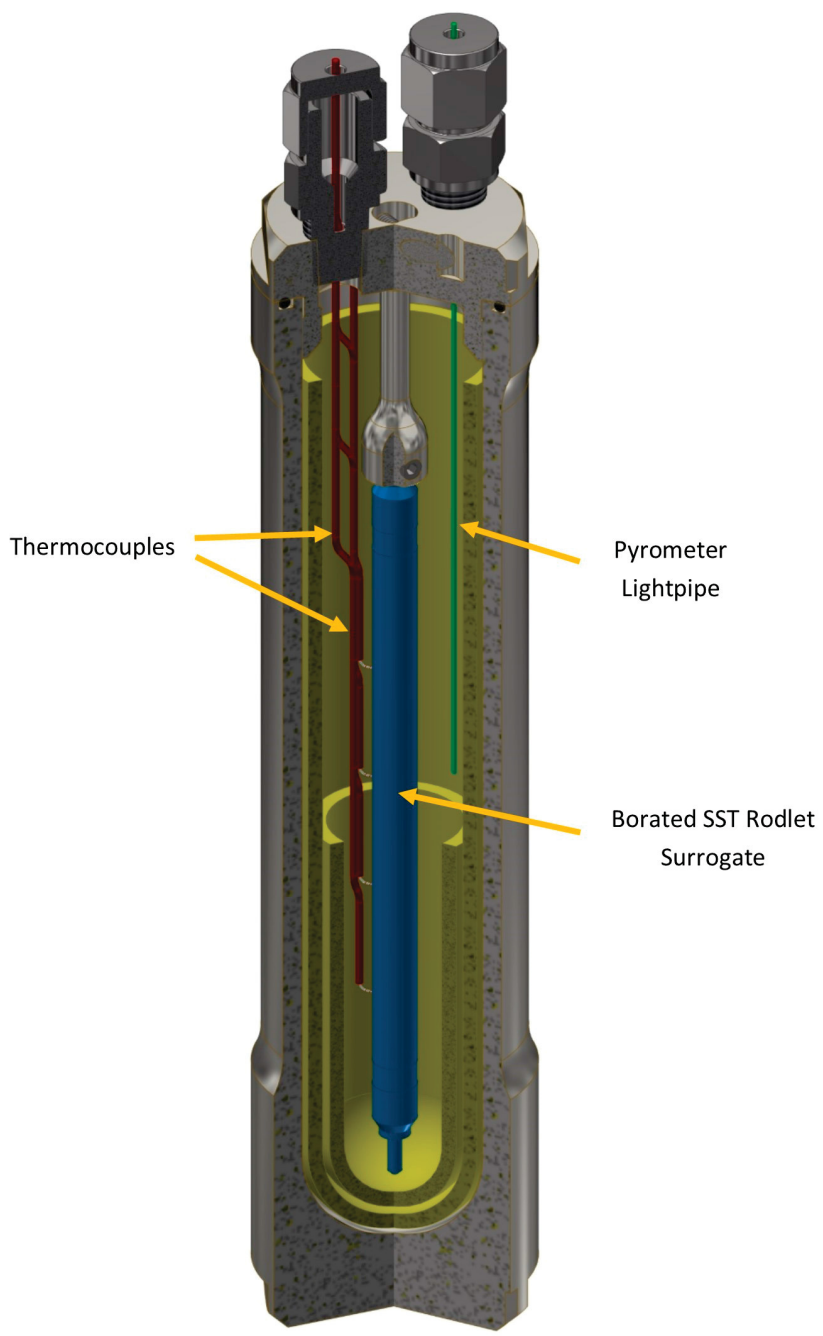

Figure 13: Example SETH Capsule with Surrogate Specimen and Temperature Instrumentation

A subtle distinction is provided here in referring to these deployment tests as either qualification tests or property measurement tests. Neither category is mutually exclusive, but is generally meant to illustrate that lower cost qualification tests may be accomplished with the use of surrogates, where property measurement tests involve end-use specimens (e.g. fuel) in a way that also has intrinsic scientific value beyond the demonstration of instrument performance alone. It is generally thought that the NEET in-pile sensor development program has the responsibility to support qualification tests, while the cross-cutting data needs represented by property measurements tests may fall under the purview of this NEET program, fuel development programs, or a combination thereof. Regardless, the conceptualized TREAT instrument irradiation capability described herein supports these progressions in any case.

Like the surrogate-based qualification tests summarized above, fuel-testing modules designed for the MARCH system can also be used for property measurement tests on end use specimens. Here the relative ease in placing instruments and fuel specimens in the MARCH system enable property measurements under neutron/gamma bombardment. This relatively under-addressed field is ripe with data opportunities that can highlight the major impact of in-situ instrumentation and advance the state of knowledge for modeling and developing nuclear fuels. In cases where these data are needed for irradiated fuels/materials, shielded hot cells at the neighboring MFC can circumvent the traditional problem of longterm instrument survival in a steady-state reactor by outfitting highly-irradiated specimens with instruments prior to in-situ property measurements in TREAT. Examples of this type of testing are shown below: 
- An adaptation of the SETH module, which makes use of temperature instrumentation and TREAT's transient shaping capability to oscillate nuclear heating in fuel specimens to determine parameters such as pellet radial thermal conductivity

- Optical fiber-based distributed measurement systems to determine cladding strain using rodlets in the SETH capsule

- Fuel axial expansion measurements using LVDTs or other sensor technologies in the THOR capsule (these data could also be compared to hodoscope data) 


\section{References}

[1] John D. Bess, Mark D. DeHart, "Baseline Assessment of TREAT for Modeling and Analysis Needs", INL/EXT-15-35372, Idaho National Laboratory, September 2015.

[2] N.E. Woolstenhulme and J.D. Wiest, “ATF Transient Testing Pre-Conceptual Design and Engineering Considerations Summary,” INL report INL/EXT-13-29898, August 2013.

[3] INL Document SAR-420, “TREAT Facility FSAR", rev 1, 03/01/2017.

[4] B.C. Gneiting, "Summary Report of the HEDL Reference Fuel TREAT Tests," HEDL-TME 83-30, February 1984.

[5] R. C. Liimatainen, R.O. Ivins, M.F. Deerwater, and F.J. Testa, "Studies of Metal-Water Reactions at High Temperatures II. TREAT Experiments: Status Report on Results with Aluminum, Stainless Steel-304, Uranium, and Zircaloy-2," ANL-6250, January 1962. 